\title{
The role of HoxA11 and HoxA13 in the evolution of novel fin morphologies in a representative batoid (Leucoraja erinacea)
}

(a) CrossMark

\author{
Shannon N. Barry and Karen D. Crow
}

\begin{abstract}
Background: Batoids exhibit unique body plans with derived fin morphologies, such as the anteriorly expanded pectoral fins that fuse to the head, or distally extended anterior pelvic fin lobes used for a modified swimming technique utilized by skates (Rajidae). The little skate (Leucoraja erinacea), exhibits both of these unique fin morphologies. These fin modifications are not present in a typical shark body plan, and little is known regarding the mechanisms underlying their development. A recent study identified a novel apical ectodermal ridge (AER) associated with the development of the anterior pectoral fin in the little skate, but the role of the posterior HoxA genes was not featured during skate fin development.

Results: We present the first evidence for HoxA expression (HoxA11 and HoxA13) in novel AER domains associated with the development of three novel fin morphologies in a representative batoid, L. erinacea. We found HoxA13 expression associated with the recently described novel AER in the anterior pectoral fin, and HoxA11 expression in a novel AER domain in the anterior pelvic fin that we describe here. We find that both HoxA11 and HoxA13 are expressed in claspers, and while HoxA11 is expressed in pelvic fins and claspers, HoxA13 is expressed exclusively in developing claspers of males. Finally, HoxA11 expression is associated with the developing fin rays in paired fins.

Conclusion: Overall, these results indicate that the posterior HoxA genes play an important role in the morphological evolution of paired fins in a representative batoid. These data suggest that the batoids utilize a unique Hox code, where the posterior HoxA genes exhibit distinct expression patterns that are likely associated with specification of novel fin morphologies.
\end{abstract}

Keywords: HoxA, Skate, Fin, AER, Clasper, Hox

\section{Background}

Vertebrates exhibit an impressive array of morphological diversity, with a variety of body plan features exhibited by extinct and extant taxa, and the cartilaginous fishes are no exception. Cartilaginous fishes represent the most ancestral lineage of extant jawed vertebrates, which diverged from the bony fishes approximately 420-450 million years ago $[1,2]$, and include the holocephalans, sharks, rays and skates. Therefore, cartilaginous fishes provide an important phylogenetic context for comparing fin and limb development in gnathostomes, and understanding the genetic

*Correspondence: crow@sfsu.edu

Department of Biology, San Francisco State University, 1600 Holloway Ave, San Francisco, CA 94127, USA underpinnings for trait variation and body plan diversity [3]. Approximately half of all cartilaginous fishes exhibit a modified body plan that is dorsoventrally flattened, with large pectoral fins that expand anteriorly and fuse to the head or rostrum (Batoidea). A compelling focus of evolutionary developmental studies has been the evolution of modified appendages such as the fin to limb transition, and these studies have highlighted co-option of ancestral genetic regulatory networks (GRNs) that were present in the common ancestor of jawed vertebrates as a primary mechanism in the evolution of body plan disparity [3, 4], including novel deployments of HoxA/D patterning genes.

Hox genes are classically associated with early patterning of anterior and posterior domains during 
embryogenesis and have the intriguing property of collinear expression, in which the genes on the $3^{\prime}$ end of the cluster are expressed early in anterior domains, followed by expression of the $5^{\prime}$ genes in more restricted and posterior domains. Later in development, HoxA/D genes play an important role during fin and limb morphogenesis, and are commonly referred to as the "fin and limb building toolkit" [5]. The expression patterns of the posterior HoxD genes have been well characterized and are associated with specification of morphological disparity via anterioposterior patterning of distal domains. The $5^{\prime}$ HoxA genes specify proximal/distal domains and also have been implicated in the morphogenesis of novel features in distal domains $[5,6]$. In tetrapods, the posterior HoxA genes, HoxA11 and HoxA13, have mutually exclusive expression patterns with HoxA11 patterning the forearm (zeugopod), and HoxA13 patterning the hand (autopod) [7-9]. HoxA11 and HoxA13 expression is overlapping during pectoral fin development in shark [10], paddlefish [11, 12] and zebrafish [13], suggesting that exclusive expression domains in the autopod is a tetrapod novelty [14].

Fins and limbs develop as outgrowths of the body wall and are patterned by two signaling centers referred to as the apical ectodermal ridge (AER), which is associated with distal outgrowth, and the zone of polarizing activity (ZPA) which is associated with AP patterning [15]. The AER plays a major role in the patterning and outgrowth of fins and limbs, and removal of the AER results in the loss of distal elements in the limbs of tetrapods [16]. AER outgrowth and patterning is accomplished via interactions with major signaling pathways involving $W n t 3$ and fibroblast growth factor 10 (Fgf10) [16, 17]. During fin development in ray-finned fishes, the AER flattens into an apical fold (AF) that will eventually give rise to the dermal fin rays [18]. However, in tetrapods, development of the AF is delayed, resulting in continued AER signaling and distal elongation of endoskeletal elements including the eventual development of digits [19]. Wnt3 is an established AER marker and is associated with underlying Fgf signaling, along with bone morphogenetic proteins (BMP) and Notch1 [20, 21]. These genes maintain the AER within the developing fin and limb mesenchyme $[17,22]$. For example, during early pectoral fin development of the little skate, the distal AER is maintained by the presence of Wnt 3 and Fgf10, along with expression of the $5^{\prime}$ HoxD genes [20, 23].

The establishment of novel AER domains is associated with the evolution of novel fin/limb morphologies. In addition to the extended AER domain promoting endoskeletal outgrowth in tetrapods, a novel AER domain was recently described in the anterior pectoral fin of the little skate that is associated with a novel morphology including anterior expansion and fusion to the pharyngeal basket, head, and rostrum [20]. Nakamura et al. [20] demonstrated that there is an additional and distinct AER in the anterior region of the little skate pectoral fin that is associated with anterior elongation by the presence of outgrowth AER markers Wnt3, Fgf7, and $3^{\prime}$ Hox genes. O'Shaughnessy [24] demonstrated another novel AER that is associated with development of claspers in the little skate [24]. Claspers are a sexually dimorphic modification to the pelvic fins of male cartilaginous fishes that develop into rolled structures extending from the medial portion of the pelvic fins and are used for sperm transfer during copulation [3,25]. A recent investigation into the development and outgrowth of claspers examined the genetic pathway driving clasper development [24]. This study found that expression of the AER marker Fgf8, in conjunction with Sonic hedgehog (Shh) pathway (regulated by Gremlin1 and Androgen Receptor) was sufficient to initiate clasper development. Further, expression of HoxD12 and HoxD13 were expressed as sex-specific genes in claspers (i.e., expressed in claspers of males but not in pelvic fins of females). However, the posterior HoxA genes were not investigated, and their role in novel morphologies such as the batoid pectoral fin and male claspers remain undescribed.

Here, we describe novel expression patterns for the posterior HoxA genes, HoxA11 and HoxA13, during the development of modified fin structures in the little skate. Distinct morphologies in the anterior pectoral and pelvic fins appear to be specified by different HoxA genes, and we propose a novel AER in anterior pelvic fin. We also found evidence suggesting that HoxA11 is associated with the development of the fin rays in the little skate, which contrasts with the role of HoxA13 in fin ray development in ray-finned fishes [26]. Finally, while HoxA11 is expressed in a broad domain marking the posterior half of the pelvic fin in males and females, HoxA13 is expressed exclusively in the posterior claspers of males in the little skate (Leucoraja erinacea), a representative batoid, which is somewhat reminiscent with limb patterning in the zeugopod and autopod of tetrapods, but without exclusion of HoxA11 by HoxA13.

\section{Methods}

\section{Embryos and staging}

Little skate embryos were obtained from the Marine Biological Laboratories (Woods Hole, MA). Embryos were removed from their egg capsules and euthanized by cervical transection. Whole embryos were placed into $4 \%$ paraformaldehyde overnight at $4{ }^{\circ} \mathrm{C}$ and then transferred to $100 \%$ methanol and stored at $-20^{\circ} \mathrm{C}$. Embryos were sexed and staged following the staging scheme of Maxwell et al. [27] for the winter skate (Leucoraja 
ocellata). Gene expression patterns were evaluated at several stages spanning paired fin development, which were stages 27, 28, 29 30, 31, 32 and early 33 (Fig. 1). A total of 205 embryos were used for this study, with 23 of those embryos utilized for clearing and staining and the remainder used for in situ hybridization.

\section{Clear and stain}

Little skate embryos at stages 30, 31, 32 and 33 were cleared and stained following Gillis et al. [3]. In short, embryos in $100 \%$ methanol were re-hydrated in gradients of ethanol (100\%;70\% at $2 \mathrm{~h}$ each), then left overnight in $0.2 \%$ alcian blue in ethanol containing $30 \%$ glacial acetic acid. Embryos were then moved to 100\% ethanol with $30 \%$ glacial acetic acid for $24 \mathrm{~h}$, followed by 3 days of ethanol gradients $(70 \% ; 50 \% ; 25 \%)$ and $24 \mathrm{~h}$ of submersion in distilled water. An addition of a $0.5 \%$ alizarin red in $\mathrm{KOH}$ was added for $24 \mathrm{~h}$ submersion, followed by de-staining in $0.5 \%$ $\mathrm{KOH}$ for up to $24 \mathrm{~h}$, and a graded glycerol series for 3 days in $0.5 \% \mathrm{KOH}$ before photographing in $80 \%$ glycerol.

\section{Whole-mount in situ hybridization}

Probes for in situ hybridization (ISH) were synthesized from constructs containing the target insert for HoxA (HoxA11, HoxA13) and HoxD (HoxD11, HoxD12, HoxD13) genes (Table 1) and cloned using the pGEM-T Vector System II (Promega) and linearized using NcoI, SpeI and SphI (Promega) following Wilkinson [28]. ISH was performed following the protocol of Wilkisnson [28], with empirical optimization of bleaching time, hybridization temperature, blocking time and NBT/BCIP staining. Following staining, embryos were re-fixed in $4 \%$ paraformaldehyde and photographed in 50\% glycerol $/ 50 \%$ PBT and stored in $100 \%$ glycerol at $4{ }^{\circ} \mathrm{C}$. Wnt 3 expression images were graciously provided by Nakamura et al. [20].

\section{Sectioning}

Following in situ hybridization, embryos for sectioning were placed into $30 \%$ sucrose in $1 \mathrm{X}$ PBS overnight at $4{ }^{\circ} \mathrm{C}$. Sucrose was removed and embryos were embedded with "O.C.T." Compound (Tissue Tech), and were immediately frozen on dry ice in plastic molds. Embryos were cryosectioned at $15 \mu \mathrm{M}$.

\section{Results}

\section{HoxA13 and HoxA11 specify two novel AER-like domains in distinctly modified regions of the anterior paired fins in the little skate}

During pectoral fin development in the little skate, HoxA13 is expressed along a narrow ridge on the distal margin from the anterior tip to the distal mid fin at stage

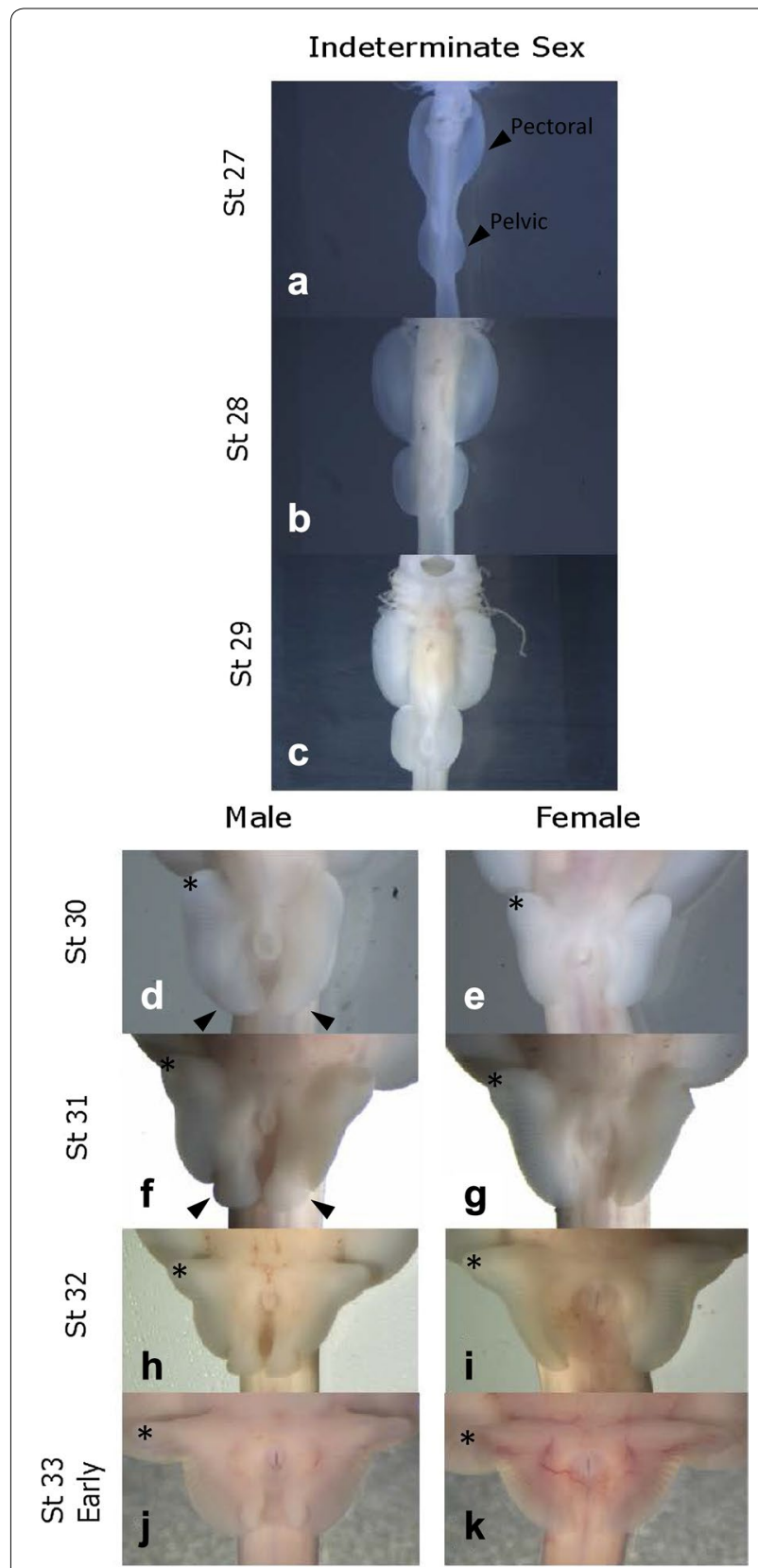

Fig. 1 Developmental staging scheme for the little skate, Leucoraja erinacea, outlining early pectoral and pelvic fin development (a-c) and later pelvic fin development (d-k). Early development shows the paired fin outgrowth from the midline of the body. Sex is indeterminate at these stages (a-c). At stage 30, clasper morphogenesis begins in males, with visible curvature of the posterior bud (d), which is absent in females (e). Outgrowth of the crura begins in the anterior pelvic fins at stage 30 as well $(\mathbf{d}, \mathbf{e})$. As development continues the claspers form a separate lobe (f) and eventually forms a rolled structure by stage 33 (j). Likewise, the crura continue to elongate distally, forming a distinct morphology (f-k). Asterisks indicate crura on both males and females. Black arrows indicate claspers (d, f) 
Table 1 HoxA and HoxD primers used for in situ hybridization probes in this study

\begin{tabular}{lll}
\hline Gene & Forward $\left(\mathbf{5}^{\prime} \mathbf{-} \mathbf{3}^{\prime} \mathbf{)}\right.$ & Reverse $\left(\mathbf{5}^{\prime} \mathbf{-} \mathbf{3}^{\prime}\right)$ \\
\hline HoxA11 & GATGAGCGGGTTCCTTGTGGC & GGTGGAGAAGGAGACGAGTC \\
HoxA13 & GCAGGAATTTGATGGCCCAT & CACCTCTGGAAGTCGAGTCT \\
HoxD11 & GGCCAAGATTTCTCGACAGT & AGTTGACCGAAAAGTCCGTG \\
HoxD12 & CAGCTGGCAAGTCTGTCAC & TCTCCCTCTGTAAATGAAGGC \\
HoxD13 & CTGCATTTGGAGCACATCAC & CTAATGGCTGGAATGGTCAAG \\
\hline
\end{tabular}

29 (Fig. 2c). Yet at stage 30, HoxA13 expression is concentrated in the anterior pectoral fin (Fig. 2d) in a region that has been described as a novel AER based on Wnt3 expression [20]. While expression of $3^{\prime}$ anterior markers HoxA2-5 in this region [20] is consistent with classic collinear expression, it was somewhat surprising to find expression of the $5^{\prime}$ HoxA13 gene in the anterior pectoral fin. Wnt3 exhibits a similar progression with expression along the entire distal margin of the pectoral fin at stage 29 , which is then restricted to the anterior pectoral fin at stage 30. By stage 30, HoxA13 shares an identical expression domain as $W n t 3$ in the region of distal outgrowth associated with batoid fin morphogenesis. HoxA13 is not expressed anywhere else in the developing pectoral fin (Fig. 2); therefore, HoxA13 expression appears to be exclusively associated with the previously described novel AER domain in little skate pectoral fin.

Interestingly, there is another outgrowth domain in the anterior pelvic fin of the little skate that elongates during morphogenesis, which is distinct from the distal outgrowth that occurs in the anterior pectoral fin. The anterior lobes of the pelvic fin, known as the crura (crus when referring to a singular lobe), are elongated into a "butterfly" shape [29] in the little skate beginning at stage 30 (Fig. 1d, e) [27]. It is likely that this region corresponds to an additional distinct AER based on Wnt3 expression at stage 30 (Fig. 2f). Wnt3 is expressed along the entire distal rim of the pelvic fin at stage 29 , but by stage 30 was restricted to the anterior pelvic fin corresponding to the crura, and the posterior region of the pelvic fin. In the anterior pelvic fin, HoxA11 expression overlaps with Wnt3 expression along a narrow ridge on the distal margin.
HoxA11 expression begins at stage 28, before outgrowth of the crura (Fig. 3a), with peak expression of HoxA11 in the elongating crura at stage 30 (Fig. 3c, d). HoxA11 expression continues until developmental stage 31 (Fig. 3e, f), as outgrowth in the crura continues to form a distinctly elongated lobe (Fig. 1f-k).

\section{The posterior HoxA genes are also expressed in broad domains marking distinct regions during pelvic fin development}

In pelvic fins of the little skate, HoxA13 expression is specific to a region corresponding with the early developing claspers (Fig. 1d-k), suggesting a role for specifying this novel domain in ancestral jawed vertebrates. Wnt3 is expressed along the distal ridge of the posterior pelvic fins, but it is unclear if this is sex specific (Fig. 2e, f). O'Shaughnessy et al. [24] described a novel AER in the developing clasper bud as indicated by sustained Fgf8, Grem 1 and Shh expression along the posterior outgrowth region of the claspers, that remained present in males well after expression had subsided in posterior pelvic fin in females (which lack claspers). We found HoxA13 expression occurs exclusively in developing claspers of males $(N=15)$, with no expression in pelvic fins of females $(N=10)$ in the little skate at stages 30-32 (Fig. 4). Further, HoxA13 is expressed in the region of the clasper anlagen in half of the embryos evaluated at stages 28 and 29 ( $n=4$ st. $28 ; n=6$ st. 29$)$, suggesting sexspecific differences during the onset of clasper specification (Fig. 4a-d), with broad expression continuing in the males until developmental stage 32 (Fig. 4e-j).

HoxA11 displays another interesting and unique expression pattern in developing pelvic fins, with a broad expression domain that marks the posterior half of the pelvic fin at stage 29 (Fig. 3a). This expression pattern later recedes to the posterior pelvic fin in both males and females by developmental stage 30 (Fig. 3c, d). This broad, posterior expression pattern of HoxA11 has not been documented previously and may be unique to batoids.

\section{HoxA11 expression is associated with fin ray development in paired fins of cartilaginous fishes}

HoxA11 marks development of fin rays, composed of cartilaginous radials that begin to develop in the proximal

\section{(See figure on next page.)}

Fig. 2 Expression of HoxA11, HoxA13 and Wnt3 in little skate pectoral and pelvic fins. HoxA11 expression is located in the anterior pelvic fin, where the crura will elongate $(\mathbf{a}, \mathbf{b})$. In the pectoral fins, HoxA13 is expressed along the distal portion of the pectoral fin, with expression restricted at the distal mid fin (c). By stage 30, HoxA13 is restricted to the anterior most portion of the pectoral fin (d). Wnt3 is broadly expressed in the entire distal domains of the pectoral and pelvic fin at stage 29 of development, but then recedes to the anterior domains of the pectoral fin and the anterior and posterior domain in the pelvic fin (e, f). Note that HoxA11 expression overlaps with Wnt3 in the anterior pelvic fins (a, b, e, f) and HoxA13 expression in the pectoral fins overlaps with the anterior Wnt3 expression (d, f), indicating that HoxA11 and HoxA13 are associated with regions of outgrowth and novel AERs. Wnt3 images are courtesy of Nakamura et al. [20]. Black arrows mark expression 


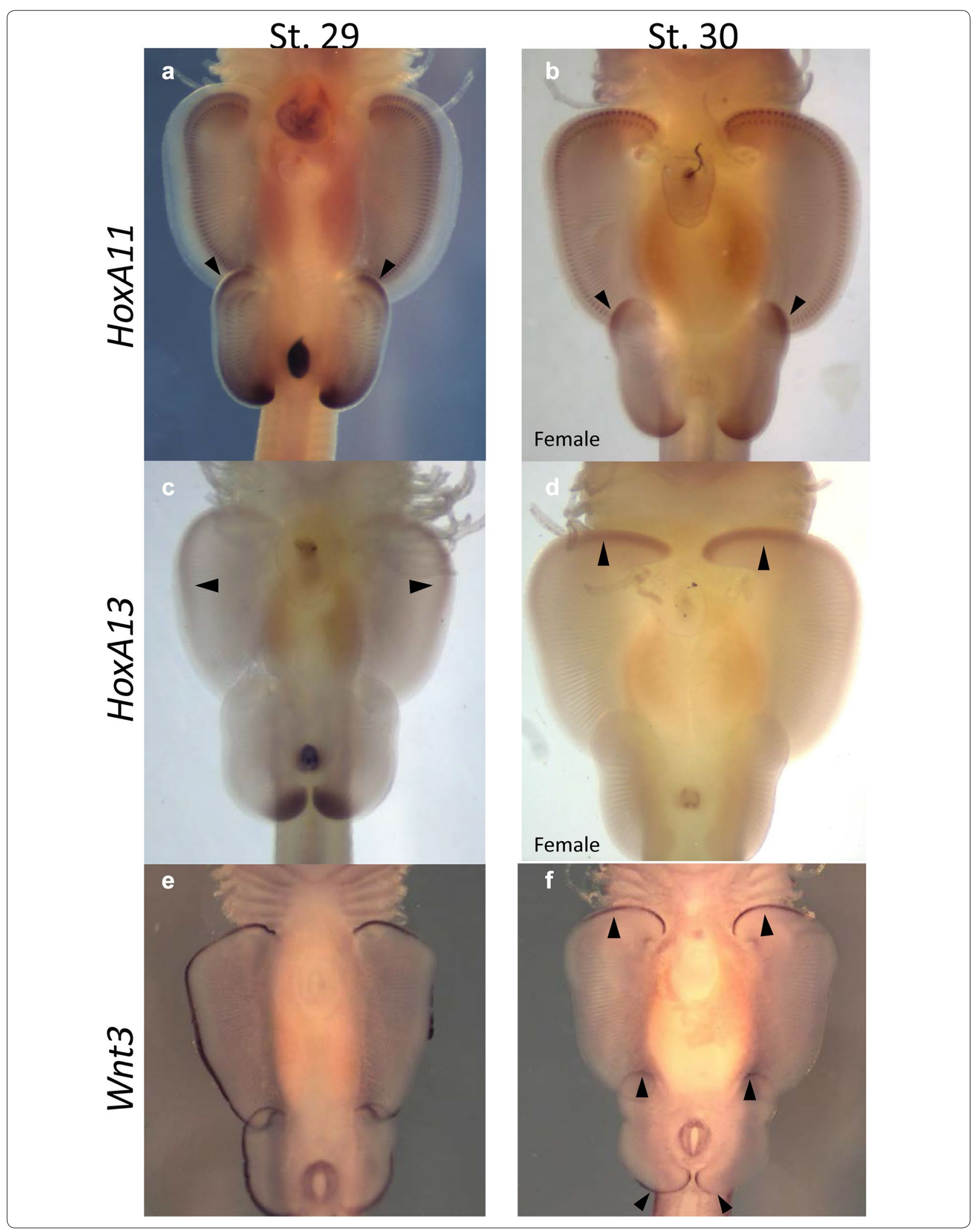




\section{$\operatorname{HoxA11}$}

\section{$\infty$ \\ N \\ in}

O

N

ن
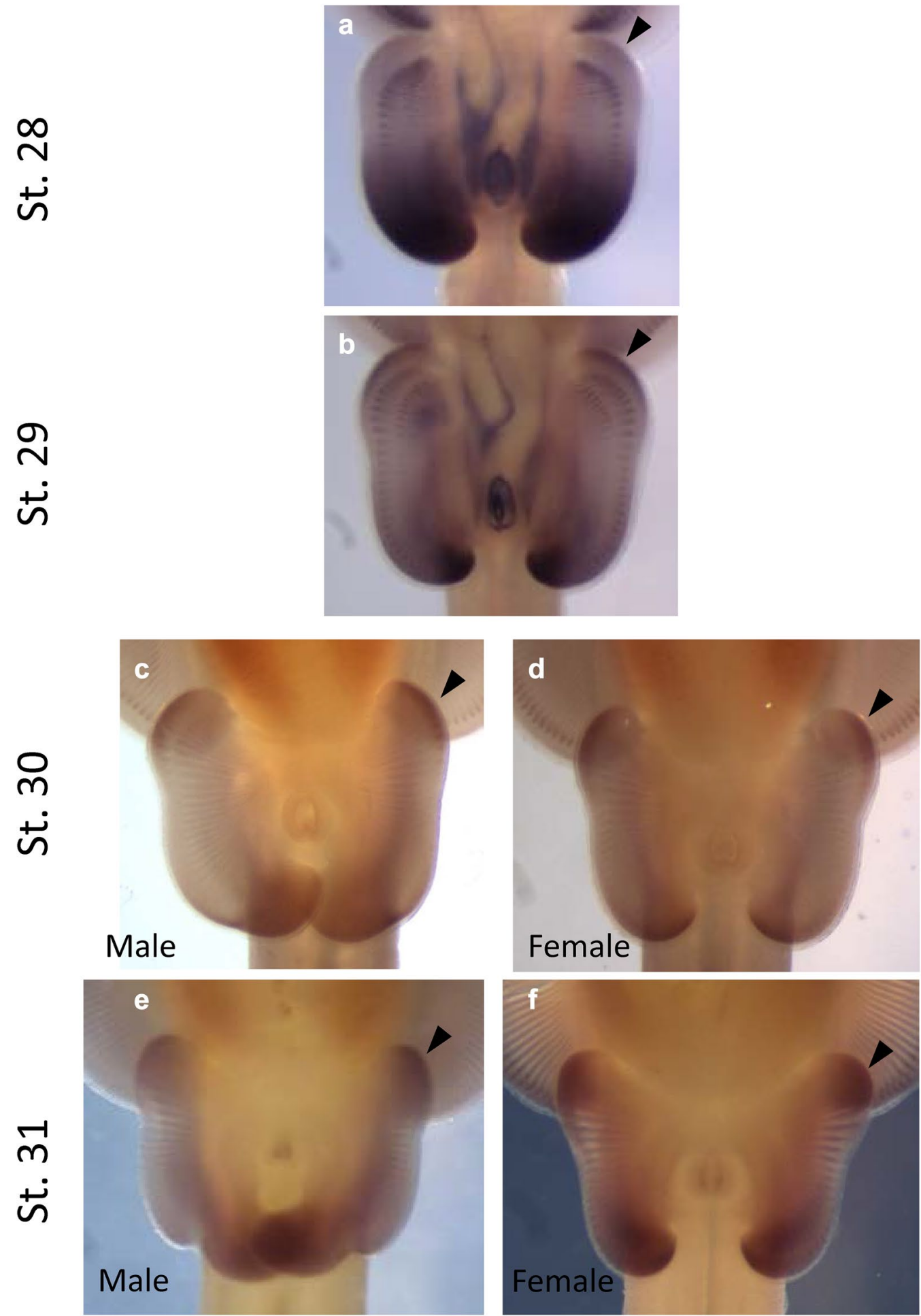

Fig. 3 HoxA11 expression in anterior pelvic fins corresponds to the developing crura. Expression is observed at stage 28 (a) and continues through stage $31(\mathbf{b}-\mathbf{f})$ in a region that overlaps with Wnt3 expression (see Fig. $2 \mathrm{~F}$ ), indicating a novel AER-like domain that is associated with the distal outgrowth of the crura. Interestingly, the crura does not begin to develop until stage 31 (e, f), indicating that HoxA11 begins to pattern the crura before elongation occurs. HoxA11 is expressed in the posterior pelvic fin in both males and females (c-f) 


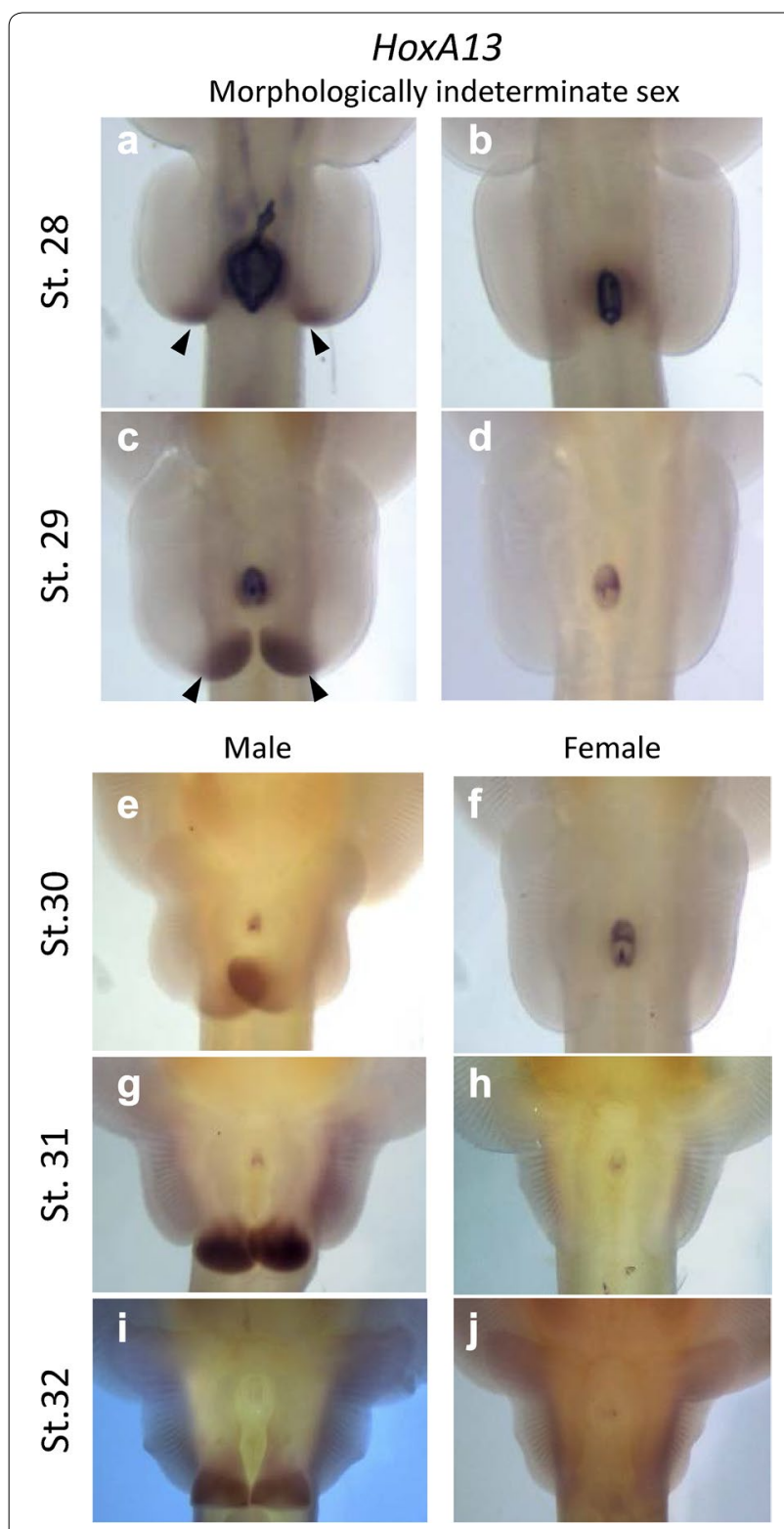

Fig. 4 HoxA13 expression is sexually dimorphic in the claspers of males, and is not expressed in pelvic fins of females. At developmental stages 28 and 29, sex cannot be determined morphologically as the claspers have not yet begun to differentiate, however HoxA13 expression occurs in half of all embryos (Black arrows $\mathbf{a}, \mathbf{c}$ ), suggesting that HoxA13 is patterning the claspers before they form (a-d). At stages 30-32, clasper morphogenesis is apparent, and HoxA13 expression continues in males, with no expression in the pelvic fins of females $(\mathbf{e}-\mathbf{j})$

finfold during early outgrowth of paired fins from the body wall (Fig. 5a). As the fins continue to expand distally, HoxA11 expression follows the distal leading edge of the fin ray elements through developmental stage 31, after which HoxA11 expression in the fin rays dissipates (see Additional file 2). We compared the number of fin ray elements in embryos of the little skate (by clear and stain specimens), and adults (by radiograph specimens) with bands of HoxA11 expression in the developing pectoral fins (Fig. 6). We were able to distinguish 53-62 bands of HoxA11 expression at stages 28-31, which is approximately ten fewer than the total number of elements in mature fins. However, it was difficult to count expression bands precisely on the anterior and posterior fin boundaries where expression bands merged or pelvic fins overlapped; therefore, the number of HoxA11 bands was likely underestimated (Fig. 6). Nonetheless, it appears that the patterning of fin rays is preceded by HoxA11 expression before development of cartilaginous condensations, as HoxA11 is no longer expressed when fin rays mature. For example, at stages 30 and 31 there are over 55-59 bands of HoxA11 expression, but only 39-43 fin rays in the mid pectoral fin have cartilaginous condensations visible by clear and stain at that stage. Therefore, chondrification of fin rays lags behind HoxA11 expression and the number of fin rays visible by these two different methods is out of sync. Remarkably, the number of fin ray elements in adults is similar to the number of cartilaginous condensations in pre-hatch embryos, with 60-70 elements clearly visible from stage 32 through adulthood (Fig. 6).

At stages 28 and 29 (Fig. 5e, Additional file 2A, B), HoxA11 expression is observed in dorsal and ventral planes of the paired fins (i.e., illustrated expression is not offset in a proximal-distal pattern but is actually expressed in overlapping dorsal and ventral planes). We sectioned the pectoral fin of embryos after whole-mount in situ hybridization, which confirmed that HoxA11 is expressed in two planes (Fig. 5f). However, sectioning of cleared and stained fins at a later stage of development revealed that cartilaginous condensations develop in the middle mesenchymal tissue of the fin, not on the dorsal and ventral planes where HoxA11 is expressed, suggesting that HoxA11 specifies regions for fin ray development or marks domains between fin rays, in a dorsal/ventral fashion (Fig. 5f, h).

\section{Discussion}

Expression of HoxA13 and HoxA11 is associated with novel AERs during paired fin morphogenesis in a representative batoid

In the pectoral fin, HoxA13 is expressed as a narrow stripe along the anterior ridge that corresponds to a novel AER [20] and overlaps with Wnt3 expression. While the overlapping expression of HoxA13 and Wnt3 is indeed intriguing, there are data suggesting a functional role for HoxA13 in the anterior expansion of the pectoral fin. When little skate embryos are exposed to retinoic acid (RA) treatment, anterior expansion of the pectoral and pelvic fins fails [30], resulting in a more "shark like" morphology. During limb regeneration in Axolotl, 


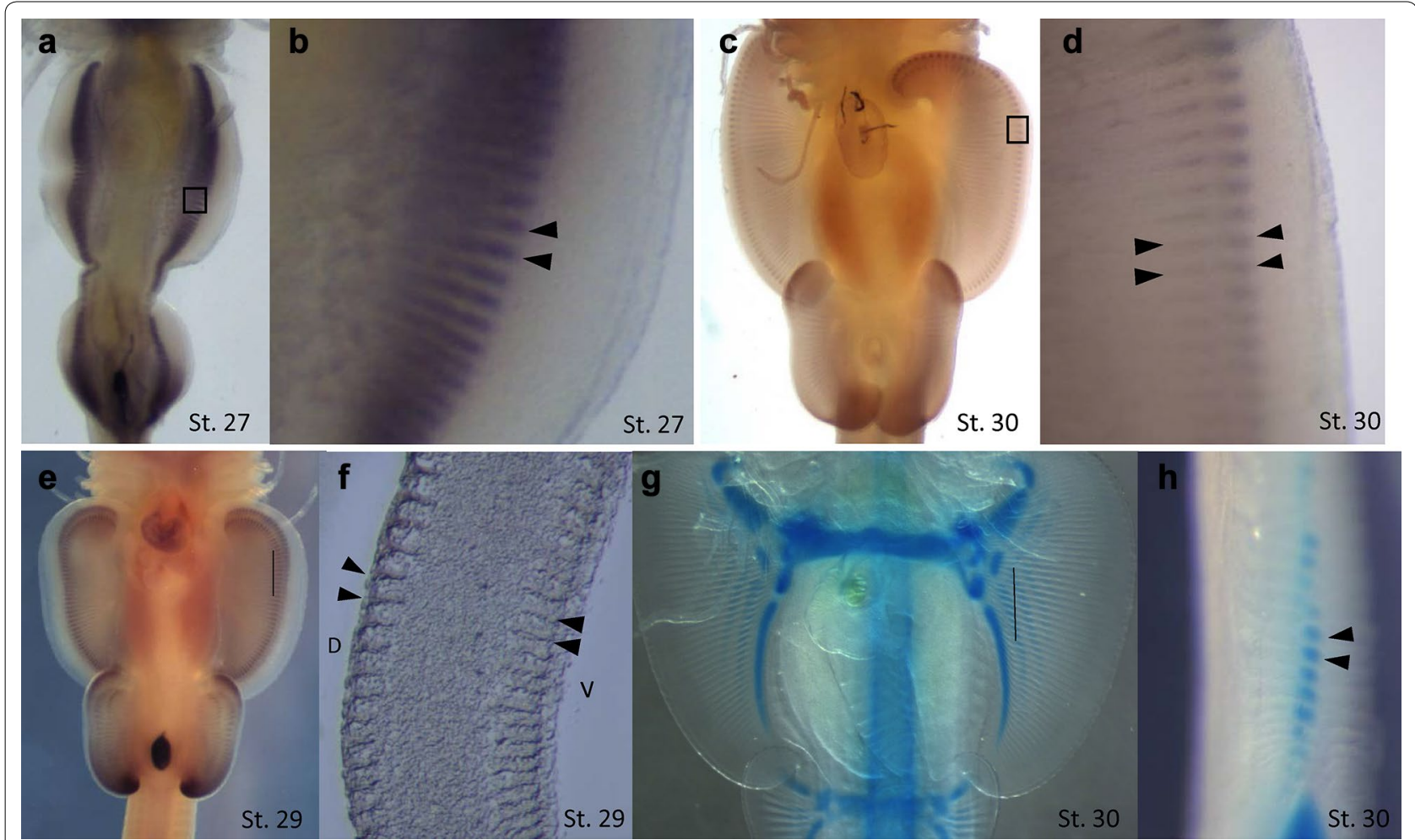

Fig. 5 HoxA11 is associated with the developing fin rays in the pectoral and pelvic fins (a-d). HoxA11 expression begins medially in paired fins as a vertical band (a) composed of horizontal stripes $(\mathbf{b})$ at developmental stage 27. As the fins grow and extends laterally, HoxA11 expression localizes distally with the fin expansion. Initially, expression is condensed with some stripes of expression merged (a), yet by stage 30, expression is associated with individual fin rays (c). While it appears that fin ray expression is in two waves, it is actually expressed in two planes, with the more distal expression on the dorsal side of the fin, and the inner expression is on the ventral side of the fin (d). Boxes indicate magnified regions. Sectioning the pectoral fin at stage 29 reveals that HoxA11 expression is not localized in the fin rays per se, rather expression occurs in dorsal and ventral domains marking segments associated with fin ray condensations $(\mathbf{e}, \mathbf{f})$. A lateral section of the pectoral fin in a cleared and stained specimen indicates that the cartilaginous condensation of the fin ray occurs in the mid-mesenchymal tissue of the developing fin ( $\mathbf{g}, \mathbf{h})$. Black arrows indicate HoxA11 expression in the developing fin rays $(\mathbf{b}, \mathbf{d}, \mathbf{F})$, and cartilaginous condensation $(\mathbf{h})$. Black lines indicate where the sections were taken $(\mathbf{e}, \mathbf{g})$. $D=$ dorsal, $V=$ ventral $(\mathbf{f})$

the majority of targets directly affected by RA signaling were those in the HoxA cluster, including HoxA11 and $\operatorname{HoxA13}$ [31]. RA functions by binding to multiple receptors (RARs/RXRs) before binding to retinoic acid response elements (RAREs) in the regulatory regions of target genes [32]. Not surprisingly, a RARE has been identified upstream of the HoxA cluster in zebrafish and mice [33].

In addition to demonstrating that HoxA13 expression is associated with a novel AER in the anterior pectoral fin (Fig. 2c, d), we also provide evidence that HoxA11 expression is associated with a morphological adaptation in anterior pelvic fin (Fig. 2a, b). Skates are adapted to benthic lifestyles, with elongated anterior pelvic fin lobes, called crura, that are utilized for an alternative mode of locomotion known as "punting", which is thought to be a more energy efficient mode of locomotion along the sea floor [29, 34]. We propose that the region corresponding to the crura is specified by an additional novel AER-like domain based on Wnt3 expression that was identified but not highlighted in Nakamura et al. [20]. Fin expansion and elongation in both the anterior pectoral and pelvic fins failed when exposed to the WNT inhibitor IWR1 [20] supporting the role of AER outgrowth via Wnt3 expression in the morphological evolution of both distinct anterior fin domains in the little skate. While O'Shaughnessy et al. [24] described expression of several genes in the Shh pathway that are associated with clasper development in the posterior pelvic fins of males, they did not discuss expression in the anterior pelvic fin associated with the outgrowth of the crura. However, a few of these genes, Grem1, Ptch1, and Shh appear to be expressed in this anterior domain. One possibility is that RA treatment could interrupt the Shh pathway, and therefore affect HoxA expression indirectly. However, Shh is not expressed in anterior pectoral fin in the little skate at stages 29-30, ([20] and pers. communication Jeff Klomp), and Grem 1 expression is biased toward the 
posterior pectoral fin [20]. Therefore, failure of anterior development in both pectoral and pelvic fins after exposure to RA [30, 35] suggests a more direct link between RA and HoxA, and is consistent with a functional role for HoxA13 and HoxA11 in specifying novel morphologies in anterior paired fins. These observations are consistent with the idea that derived lineages that have undergone fin and limb modifications exhibit novel expression patterns of HoxA11 and HoxA13 that are associated with development of unique features, such as the proximo-distal expression pattern in the zeugopod and autopod of tetrapods, and the extended anterior paired fins of batoids as selective advantages for different habitats and life histories. While there appear to be many novel AERs associated with these outgrowths and modifications, each is associated with a unique HoxA code that may be associated with domain specification and identity.

\section{HoxA11 and HoxA13 mark broad domains in posterior regions of fin/limbs with novel morphologies}

HoxA13 expression occurs in a broad domain that is exclusive to the developing claspers. Claspers begin to bud from the posterior pelvic fin at developmental stage 30 , meaning that earlier stages of the little skate cannot be sexed. Yet differences were noted in $\operatorname{Hox} A 13$ expression prior to clasper morphogenesis, with half the embryos displaying HoxA13 expression at stages 28 and 29 (Fig. 4a-d), suggesting that HoxA13 sets up early patterning of the claspers. The posterior HoxD genes, HoxD12 and HoxD13, are also sexually dimorphic, with no expression in females, and expression domains restricted to the developing claspers (see Additional file $1 \mathrm{~A}-\mathrm{H}$ ) [24]. There was no HoxA13 expression in the posterior regions of developing pectoral or pelvic fins in the little skate, contrary to what has been observed in the fins of catshark [10], zebrafish [13] and paddlefish [11], and the limbs of frog [7] and chick [8], suggesting that altering the Hox code in specific lineages is associated with specific novel morphologies.

HoxA11 is expressed in both males and females during pelvic fin development (Fig. 3c-f), with expression in the anterior region of the crura, and the posterior half of the pelvic fin, including the claspers of males. The broad expression domain of HoxA11 in posterior pelvic fins may be unique to batoids. However, HoxA11 also has a broad expression domain in the paddlefish pectoral fin bud [11], and in the forearm (zeugopod) in tetrapods that is excluded in the distal autopod by HoxA13

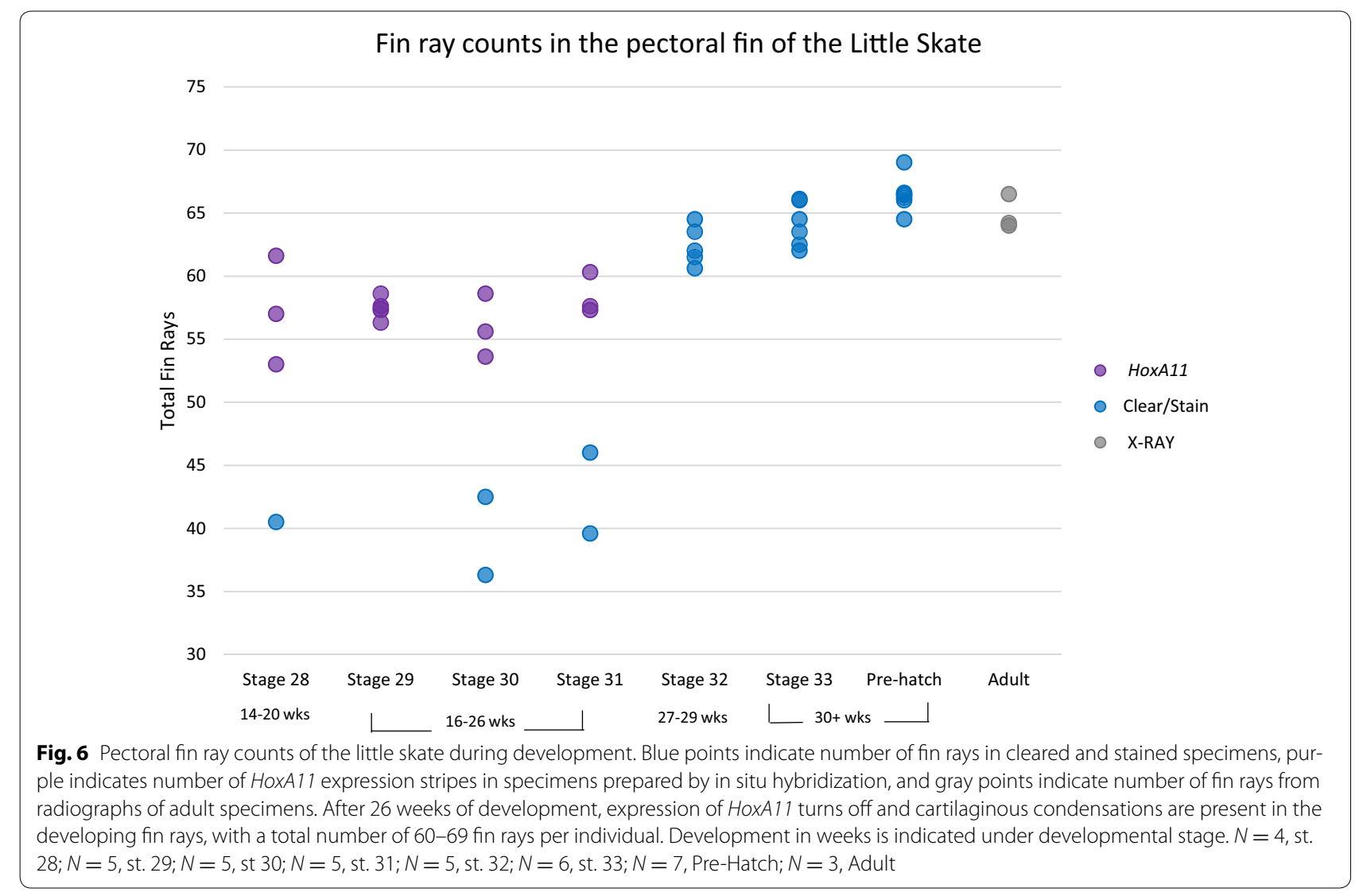


$[9,36,37]$. It is worth noting that tetrapod forelimbs are homologous with the posterior region of paired fins (metapterygium or basipterygium) in cartilaginous fishes. Regardless, we observe a broad expression domain of HoxA11 in the posterior pelvic fin/clasper, while HoxA13 exhibits a broad expression domain that is specific to the developing claspers. These data suggest that the specific expression patterns of $\operatorname{Hox} A 11$ and HoxA13 are associated with the evolution and development of novel fin/limb morphologies in the zeugopod, autopod and claspers, and the role of HoxA11 in posterior pelvic fin warrants further investigation.

\section{HoxA11 is associated with development of support structures in pectoral and pelvic fins}

HoxA11 is expressed in association with the developing fin rays in pectoral and pelvic fins of the little skate. This expression pattern was also illustrated, but not described, in the developing fins of the catshark [10, 38]. In zebrafish, HoxA13 expression is associated with the developing fin rays [26], but HoxA11 was not evaluated. We found no evidence for HoxA13 expression in the fin rays in the little skate, despite strong HoxA13 expression in other areas of fin outgrowth and development. This suggests that $\operatorname{HoxA11}$ is associated with patterning of fin rays in the cartilaginous fishes, and $H o x A 13$ may have been coopted in fin ray expression in ray-finned fishes.

By comparing HoxA11 expression bands in pectoral fins with cartilaginous condensations in cleared and stained juveniles and X-rays of older specimens, we discovered that the number of fin rays is specified early in development (Fig. 6), which is preceded by stripes of HoxA11 expression along the dorsal and ventral margins of paired fins, followed by chondrification in the midmesenchymal tissue (Fig. 5e-h). We can observe what appears to be waves of cartilage that may correspond to the individual radial elements of the fin rays at stage 30 (Fig. 5g).

Interestingly, paired fins develop from continuous finfolds that extend laterally down the sides of the body in the little skate, from the region posterior to the head to the cloaca (Fig. 1). At stage 27, HoxA11 expression occurs in a continuous field along this entire domain (Fig. 5a) marking early patterning of fin rays. Yet by stage 28 , the pectoral and pelvic fins are differentiated, and HoxA11 expression and the finfold tissue between them is no longer present (see Additional file 2B). This developmental scheme is consistent with the finfold theory proposed by Thacher [39], which suggests that paired fins evolved as the "retained portions of a continuous lateral fin" on either side of the body (Mivart [40] and Balfour [41], and described in Tulenko, McCauley [42]). Freitas [43] argued that paired fins evolved from median fins based on shared HoxD expression patterns. HoxA expression patterns also inform aspects of the origin of fin/limbs such as the HoxA11 pattern we see in fin rays of the little skate, but to our knowledge HoxA expression has not been evaluated in medial fins of jawless or ancestral jawed vertebrates.

\section{Conclusions}

The batoids exhibit fin modifications that provide a unique opportunity to investigate the role of Hox genes in novel morphologies. HoxA genes are relatively understudied and we provide evidence that the posterior HoxA genes play distinct roles in two novel AERs during skate fin development, one of which is newly described here. Further, the little skate exemplifies unique Hox expression patterns differing from the catshark, including large posterior expression domains of HoxA11 in the pelvic fin (Fig. 7). HoxA11 is also associated with the development and patterning of fin rays in cartilaginous fishes, in contrast to HoxA13 


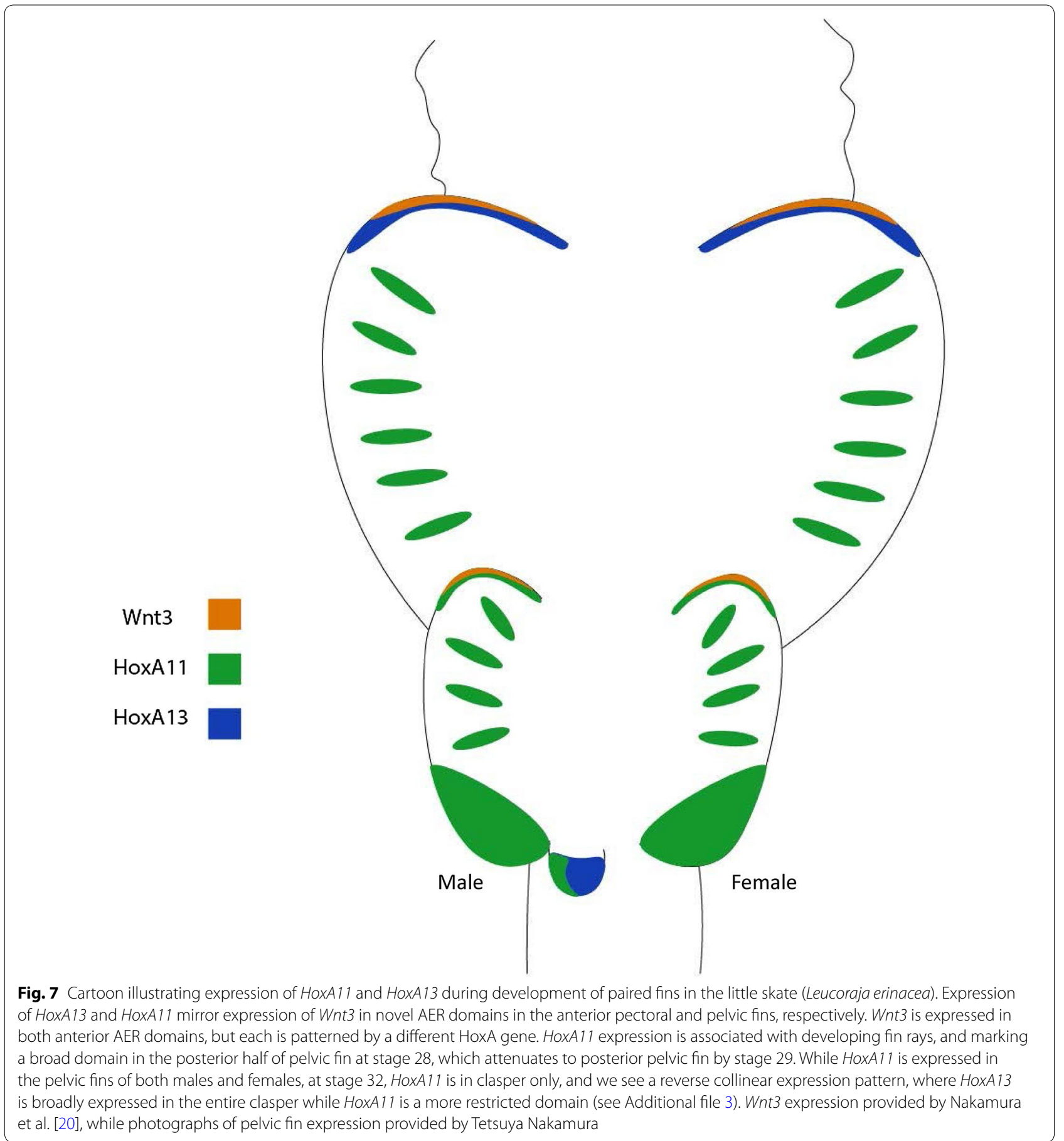

patterning fin rays in ray-finned fishes. Here, we have shown that the posterior HoxA genes appear to play a significant role in paired fin morphogenesis in batoids, and it is intriguing that each of the novel fin domains described is associated with a discrete HoxA code that is consistent with their unique specification. 


\section{Additional files}

Additional file 1. HoxD expression in the pelvic fin of the little skate during early to late development $(\mathbf{A}-\mathbf{H})$. Note that both HoxD12 (A-B) and HoxD13 (C-H) are expressed exclusively in the claspers and show no expression in the female pelvic fin. HoxD12 is expressed in the posterior pectoral fin (I), HoxD13 shows no expression at stage 30 in the pectoral fin (J). Similarly, the posterior HoxA genes are not expressed in the posterior pectoral fin, indicating a unique Hox code that specifies specific morphologies during development.

Additional file 2. HoXA11 is associated with the developing fin rays in the pectoral and pelvic fins $(\mathbf{A}-\mathbf{E})$. HoxA11 expression begins medially in the pectoral and pelvic fins in small stripes $(\mathbf{A})$. As the fins continue to elongate, HoxA11 expression elongates distally, until stage 31 which is the last-stage expression is noted $(\mathbf{E})$.

Additional file 3. Reverse collinear (RC) expression in the claspers of the little skate. RC expression occurs when the more posterior gene has a broader expression range than the gene anterior to it. In this case, HoxA13 is expressed throughout the entire clasper (B), whereas HoxA11 is restricted to the distal region $(\mathbf{A})$. This expression pattern is likely setting up left-right asymmetry in the claspers and is only observed at stage 32 of development. Arrows denote HoxA11 expression in the distal claspers.

\section{Authors' contributions}

$\mathrm{KC}$ conceived the project. SB performed all experiments. Both authors contributed to the interpretation of data and writing of manuscript. Both authors read and approved the final manuscript.

\section{Acknowledgements}

We would like to thank the Marine Biological Laboratory at Woods Hole for providing embryos and Andrew Gillis for sending initial tissue samples. We would also like to thank Tetsuya Nakamura and Neil Shubin for providing Wnt3 images. We thank Julio Ramirez for providing assistance with sectioning and Kayla Hall for counting and photographing fin rays.

\section{Competing interests}

The authors declare that they have no competing interests.

\section{Availability of data and materials}

All data generated or analyzed during this study are included in this published article.

\section{Ethics approval}

All specimens were handled and euthanized in accordance with San Francisco State University IACUC Protocol Number A14-01.

\section{Funding}

This work was supported by the following grants: The American Elasmobranch Society Student Research Award and The Dr. Earl H. Myers and Ethel M. Myers Oceanographic and Marine Biology Trust awarded to SB and NSF Awards IOS1022509 and IOS-1656487 to KC.

\section{Publisher's Note}

Springer Nature remains neutral with regard to jurisdictional claims in published maps and institutional affiliations.

Received: 11 September 2017 Accepted: 21 November 2017 Published online: 01 December 2017

\section{References}

1. Oulion S, et al. Evolution of Hox gene clusters in gnathostomes: insights from a survey of a shark (Scyliorhinus canicula) transcriptome. Mol Biol Evol. 2010;27(12):2829-38.
2. Wourms JP, Demski LS. The reproduction and development of sharks, skates, rays and ratfishes: introduction, history, overview, and future prospects. The reproduction and development of sharks, skates, rays and ratfishes. Netherlands: Springer; 1993. p. 7-21.

3. Gillis JA, Shubin NH. The evolution of gnathostome development: insight from chondrichthyan embryology. Genesis. 2009;47(12):825-41.

4. Coates MI, Cohn MJ. Fins, limbs, and tails: outgrowths and axial patterning in vertebrate evolution. BioEssays. 1998;20(5):371-81.

5. Archambeault S, Taylor J, Crow K. HoxA and HoxD expression in a variety of vertebrate body plan features reveals an ancient origin for the distal Hox program. EvoDevo. 2014;5(1):44

6. Crow KD, Amemiya CT, Roth J, Wagner GP (2009) Hypermutability of HoxA13A andfunctional divergence from its paralog are associated with the origin of a novel developmental feature inzebrafish and related taxa (Cypriniformes). Evol 63(6), 1574-92

7. Ohgo $\mathrm{S}$, et al. Analysis of hoxa11 and hoxa13 expression during patternless limb regeneration in Xenopus. Dev Biol. 2010;338(2):148-57.

8. Nelson C, et al. Analysis of Hox gene expression in the chick limb bud. Development. 1996;122:1449-66.

9. Kherdjemil Y, et al. Evolution of Hoxa11 regulation in vertebrates is linked to the pentadactyl state. Nature. 2016;539:89.

10. Sakamoto K, et al. Heterochronic shift in Hox-mediated activation of sonic hedgehog leads to morphological changes during fin development. Plos One. 2009;4(4):e5121.

11. Davis MC, Dahn RD, Shubin NH. An autopodial-like pattern of Hox expression in the fins of a basal actinopterygian fish. Nature. 2007;447(7143):473-6.

12. Metscher $\mathrm{BD}$, et al. Expression of Hoxa-11 and Hoxa-13 in the pectoral fin of a basal ray finned fish, Polyodon spathula: implications for the origin of tetrapod limbs. Evol Dev. 2005;7(3):186-95.

13. Ahn D, Ho R. Tri-phasic expression of posterior Hox genes during development of pectoral fins in zebrafish: implications for the evolution of vertebrate paired appendages. Dev Biol. 2008;322:220-33.

14. Fromental-Ramain C, et al. Hoxa-13 and Hoxd-13 play a crucial role in the patterning of the limb autopod. Development. 1996;122(10):2997-3011.

15. Burke AC, et al. Hox genes and the evolution of vertebrate axial morphology. Development. 1995;2(1):333-46.

16. Lu PF, et al. The apical ectodermal ridge is a timer for generating distal limb progenitors. Development. 2008;135(8):1395-405.

17. Mercader N. Early steps of paired fin development in zebrafish compared with tetrapod limb development. Dev Growth Differ. 2007:49(6):421-37.

18. Thorogood P. The development of the teleost fin and implications for our understanding of tetrapod limb evolution. In: Hinchliffe JR, Hurle JM, Summerbell D, editors. Developmental patterning of the vertebrate limb. Boston: Springer; 1991. p. 347-54.

19. Schneider I, Shubin N. The origin of the tetrapod limb: from expeditions to enhancers. Trends Genet. 2013:29:419-26.

20. Nakamura T, et al. Molecular mechanisms underlying the exceptional adaptations of batoid fins. Proc Natl Acad Sci USA. 2015;112(52):15940-5.

21. Fernandez-Teran M, Ros MA. The apical ectodermal ridge: morphological aspects and signaling pathways. Int J Dev Biol. 2004;52(7):857-71.

22. Laufer $E$, et al. Expression of radical fringe in limb-bud ectoderm regulates apical ectodermal ridge formation. Nature. 1997;386(6623):366-73.

23. Freitas R, Zhang G, Cohn MJ. Biphasic Hoxd gene expression in shark paired fins reveals an ancient origin of the distal limb domain. PLOS ONE. 2007;2(8):e754.

24. O'Shaughnessy KL, Dahn RD, Cohn MJ. Molecular development of chondrichthyan claspers and the evolution of copulatory organs. Nat Commun. 2015;6:6698

25. LaMarca MJ. The functional anatomy of the clasper and clasper gland of the yellow stingray, Urolophus Jamaicensis (Cuvier). J Morphol. 1964;114(2):303-23.

26. Nakamura T, et al. Digits and fin rays share common developmental histories. Nature. 2016;537(7619):225-8.

27. Maxwell EE, Fröbisch NB, Heppleston AC. Variability and conservation in late chondrichthyan development: ontogeny of the winter skate (Leucoraja ocellata). Anat Rec Ad Integr Anat Evol Biol. 2008;291(9):1079-87.

28. Wilkinson DG. In situ hybridization: a practical approach, Oxford, England: Oxford University Press, IRLPress; 1992.

29. Koester DM, Spirito CP. Punting: an unusual mode of locomotion in the little skate, Leucoraja erinacea (Chondrichthyes: Rajidae). Copeia. 2003:3:553-61. 
30. Dahn RD, et al. Sonic hedgehog function in chondrichthyan fins and the evolution of appendage patterning. Nature. 2006;445:311.

31. Gardiner DM, et al. Regulation of HoxA expression in developing and regenerating axolotl limbs. Development. 1995;121(6):1731-41.

32. Schubert $\mathrm{M}$, et al. A retinoic acid-Hox hierarchy controls both anterior/ posterior patterning and neuronal specification in the developing central nervous system of the cephalochordate amphioxus. Dev Biol. 2006;296(1):190-202.

33. Langston AW, Thompson JR, Gudas LJ. Retinoic acid-responsive enhancers located $3^{\prime}$ of the Hox A and Hox B homeobox gene clusters. Functional analysis. J Biol Chem. 1997;272(4):2167-75.

34. Macesic LJ, Summers AP. Flexural stiffness and composition of the batoid propterygium as predictors of punting ability. J Exp Biol. 2003;215(12):2003-12.

35. Gillis JA, Dahn RD, Shubin NH. Shared developmental mechanisms pattern the vertebrate gill arch and paired fin skeletons. Proc Natl Acad Sci. 2009;106(14):5720-4.

36. Zakany J, Duboule D. The role of Hox genes during vertebrate limb development. Curr Opin Genet Dev. 2007;17(4):359-66.
37. Woltering J, et al. Conservation and divergence of regulatory strategies at Hox Loci and the origin of Tetrapod digits. PLoS Biol. 2014;12:e1001773.

38. Tulenko FJ, et al. Fin-fold development in paddlefish and catshark and implications for the evolution of the autopod. Proc R Soc B. 1855;2017(284):20162780.

39. Thacher JK. Median and paired fins, a contribution to the history of vertebrate limbs. Trans Conn Acad Sci. 1877:3:281-308.

40. Mivart SG. Notes on the fins of elasmobranchs, with considerations on the nature and homologues of vertebrate limbs. Trans Zool Soc Lond. 1879;10(10):439-84

41. Balfour F. On the development of the skeleton of the paired fins of Elasmobranchii, considered in relation to its bearing on the nature of the limbs of the vertebrata. Proc Zool Soc Lond. 1881;49(3):656-71.

42. Tulenko FJ, et al. Body wall development in lamprey and a new perspective on the origin of vertebrate paired fins. Proc Natl Acad Sci USA. 2013;110(29):11899-904.

43. Freitas R, Zhang GJ, Cohn MJ. Evidence that mechanisms of fin development evolved in the midline of early vertebrates. Nature. 2006;442(7106):1033-7.

\section{Submit your next manuscript to BioMed Central and we will help you at every step:}

- We accept pre-submission inquiries

- Our selector tool helps you to find the most relevant journal

- We provide round the clock customer support

- Convenient online submission

- Thorough peer review

- Inclusion in PubMed and all major indexing services

- Maximum visibility for your research

Submit your manuscript at www.biomedcentral.com/submit 